\title{
PSA Progression
}

National Cancer Institute

\section{Source}

National Cancer Institute. PSA Progression. NCI Thesaurus. Code C123611.

The amount of prostate specific antigen is increased, based on pre-defined thresholds. 